\title{
SILICENE: \\ SILICON CONQUERS THE 2D WORLD
}

- Guy Le Lay*, Eric Salomon, Thierry Angot - DOI: 10.1051/epn/2016101

- Aix-Marseille University - Physique des Interactions loniques et Moléculaires - UMR7345

Avenue Escadrille Normandie Niemen - 13013 Marseille, France - * guy.lelay@univ-amu.fr

We live in the digital age based on the silicon chip and driven by Moore's law. Last July, IBM created a surprise by announcing the fabrication of a $7 \mathrm{~nm}$ test chip with functional transistors using, instead of just silicon, a silicon-germanium alloy. Will silicon be dethroned?

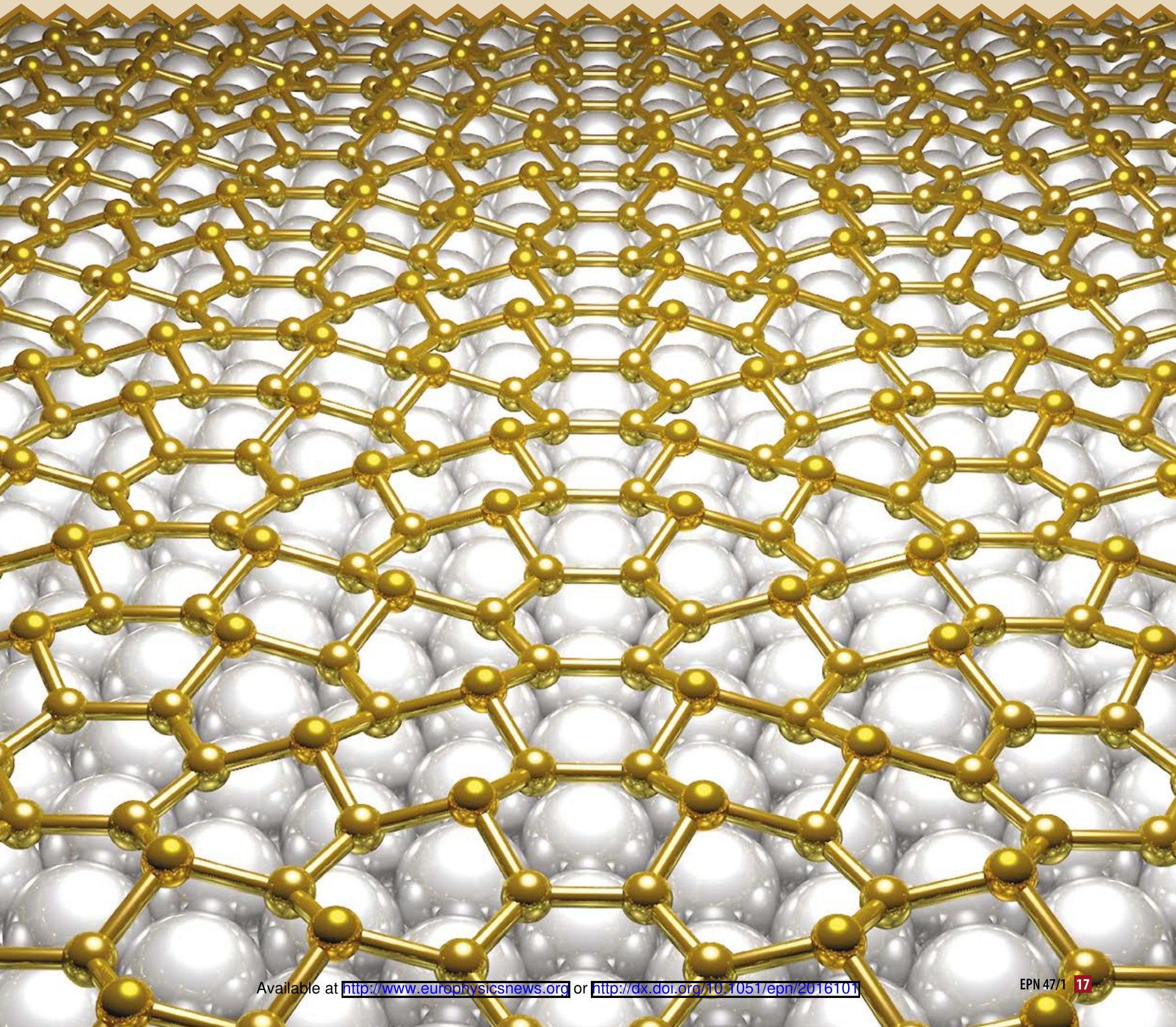




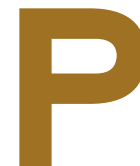

rogress in electronics is governed by the motto "smaller, faster, cheaper" and still follows Moore's law, which predicted that the number of transistors in a semiconductor chip roughly doubles every 18 months. Indeed, the key electronic device, the field-effect transistor (FET), has been shrinking continuously. Yet, the characteristic length of the channel, presently $14 \mathrm{~nm}$ for Intel's most advanced commercial central processing units (CPUs), is approaching the limits where three-dimensional (3D) silicon would be problematic. The $7 \mathrm{~nm}$ $\mathrm{Si}$-Ge transistors will enable at least $50 \%$ power/performance increase over most advanced $10 \mathrm{~nm}$ technology planned by Intel for 2016, especially by improving the mobilities of the carriers. Now the next step, the 5 nm node, appears at hand, which raises a fundamental question: at such a small nanoscale, what will be the next silicon?

\section{The end of the silicon age?}

The advent in 2004 of graphene [1], the two-dimensional (2D) material by excellence, descendent from its parent crystal graphite, was celebrated by the Nobel Prize in physics in 2010, awarded to Andre Geim and Konstantin Novoselov. It has prompted enthusiasts of this "wonder material" to predict a new carbon-based electronics era.

However, it appeared that the difficulty in opening a sizeable band gap in graphene was an insurmountable handicap for digital applications. As a consequence, the question raised by Ross Kozarsky, senior analyst at Lux Research, at the Washington DC Nanotech Conference \& Expo 2013: "Is Graphene the Next Silicon... Or Just the Next Carbon Nanotube?" was finally answered last July (2015): "Just don't expect graphene to live up to the untenable hype, or become the next silicon".

Hence, in the wake of graphene, researchers have initially looked for existing semiconductor lamellar crystals

v FiG. 1: Illustration of the buckled structure (corrugated stage) of silicene: (a) perspective view, (b) top view, (c) side view. The A and B sites, composed of Si atoms, form two sublattices which are vertically separated by $\Delta \sim 0.04 \mathrm{~nm}$.

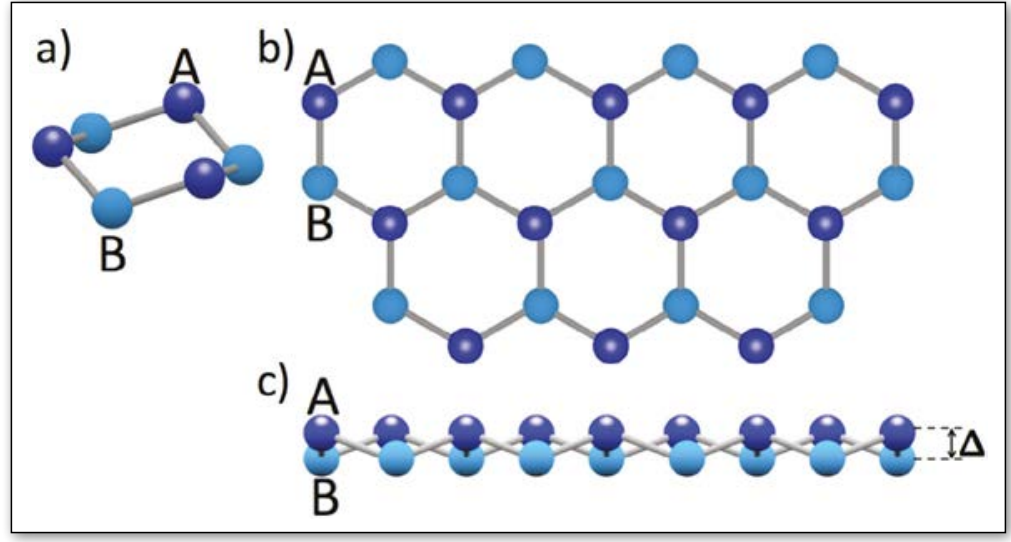

which - just as in the case of graphite - could be peeled to the ultimate limit by the famous scotch tape method. Success came in 2011 with molybdenum disulfide, which permitted the first fabrication of a transistor with a $2 \mathrm{D}$ single layer $\mathrm{MoS}_{2}$ channel [2]. However, although potentially promising for flexible electronics and for optoelectronics, such transition metal dichalcogenides transistors cannot replace advanced Si ones.

\section{The birth of silicene}

Concurrently, Patrick Vogt and Guy Le Lay (PV and GLL) in Marseille have been striving, in a creative endeavour, to synthesize silicene, the Si-based analogue of graphene. Already in an article published in 1994 [3], but essentially unnoticed for 17 years, Kyozaburo Takeda and Kenji Shiraishi had theoretically predicted its possible existence as a standalone novel $2 \mathrm{D}$ silicon allotrope with a corrugated stage, i.e., with a $\sim 0.4 \mathrm{~nm}$ out-of-plane buckling between the $A$ and $B$ sublattices, see Fig. 1. This is at variance with graphene, which is basically flat.

As underlined by Kehui $\mathrm{Wu}[4]$ and many experimentalists, the early claim in 2010 of "Epitaxial growth of a silicene sheet" with nearly planar primitive honeycomb structure and much too short Si-Si bond lengths was a false start. This illustrates the famous quote of Luigi Galvani: "for it is easy in experimentation to be deceived, and to think one has seen and discovered what one has desired to see and discover".

In fact, the first compelling experimental evidence for successful silicene synthesis on a silver (111) substrate appeared in April 2012 [5]. A significant consecutive marker has been the sudden jump in citations of Takeda and Shiraishi's paper, and of the number of articles on silicene, germanene and stanene, graphene's $\mathrm{Si}, \mathrm{Ge}$ and Sn cousins, from year 2012 on.

Another milestone is the insertion of these novel elemental artificial 2D materials - which have to be created on a template since they have no parent crystal in nature - in the EU "Science and technology roadmap for graphene, related two-dimensional crystals, and hybrid systems" [6].

Here it is worth citing one of the small but strong lessons from chemistry for nanoscience by Nobel laureate Roald Hoffmann and his silicene interlude [7]: "We have graphene multilayers and the monolayer, and the intriguing physics of these. There is a growing literature out there of the Si analogue, silicene. And that literature talks about silicene as if it were graphene. In part this is an attempt to live off graphene's mystique, but part comes out of lack of knowledge of chemistry. I don't often say something categorical, but I will say that a pristine free-standing single layer sheet of silicene (or a Si nanotube) will not be made. Silicene exists and will be made only on a support of some sort, metal or semiconductor". 


\section{Compliant single layer silicene}

Indeed, PV and GLL created, in situ, under ultra-high vacuum, single layer silicene on a silver plate, typically by exploring - like Alice - through the looking glass. Instead of depositing silver onto a piece of silicon wafer, to form an atomically abrupt prototypical noble-metal/ semiconductor interface with a "magic" epitaxial relationship (as had been done by GLL during years of research on the formation of Schottky barriers, a very hot topic in the eighties [8]) the process was inverted: they deposited silicon onto a silver (111) single crystal surface kept at $\sim 200^{\circ} \mathrm{C}$. Then, sharp low-energy electron diffraction patterns and the splendid unique "flower pattern" discovered in STM imaging and displayed in Fig. 2a were observed, revealing a highly ordered 2D epitaxial system but absolutely no direct graphene-like aspect.

The hidden underlying honeycomb structure of silicene within the "flower pattern" was unraveled thanks to high-resolution synchrotron radiation angle-resolved photoelectron spectroscopy (SR-ARPES) measurements and thorough density functional theory calculations based on an initial geometrical model inferred from the knowledge of the "magic" relationship mentioned above. Typically, a reconstructed silicene monolayer on top (with basis vectors 3 times larger than the unit vectors of free standing silicene, corresponding to a so-called $3 \times 3$ reconstruction) matched nearly perfectly a facing $4 \times 4$ supercell of the (111) silver surface below -see Fig. $2 b$. Within the $3 \times 3$ silicene lattice the Si-Si bond lengths are close to those calculated for free-standing silicene $(0.225 \mathrm{~nm}$ [9]) but the buckling is nearly doubled, increasing from $0.044 \mathrm{~nm}$ to $0.083 \mathrm{~nm}$. This is nicely confirmed by dynamic LEED measurements and, strikingly, reflection high-energy positron diffraction experiments performed in Tsukuba (Japan) [10].

The high flexibility of silicene, inherent to its intrinsic buckling associated with its intermediate $\mathrm{sp}^{2} / \mathrm{sp}^{3}$ hybridization state, makes silicene a highly compliant $2 \mathrm{D}$ material: it conforms to its substrate instead of making loose Moiré patterns, as graphene grown on metals generally does.

\section{More Moore...}

and More than Graphene!

The intrinsic low buckling in standalone silicene preserves the key electronic features found in graphene, namely the massless Dirac fermion character of the charge carriers, their extremely high mobilities and the gapless semimetal nature. Yet, symmetry breaking of the $A$ and $B$ sublattices, in addition to reconstructions, as observed until now on all metallic substrates, can eventually open up a band gap [11].

Such an opening is further facilitated by the reactivity of silicene, at variance with the inertness of graphene. As a striking example, Fig. 3 displays the ordered adsorption of atomic hydrogen, first evidence by Qiu et al. [12], which will allow further band gap tuning.
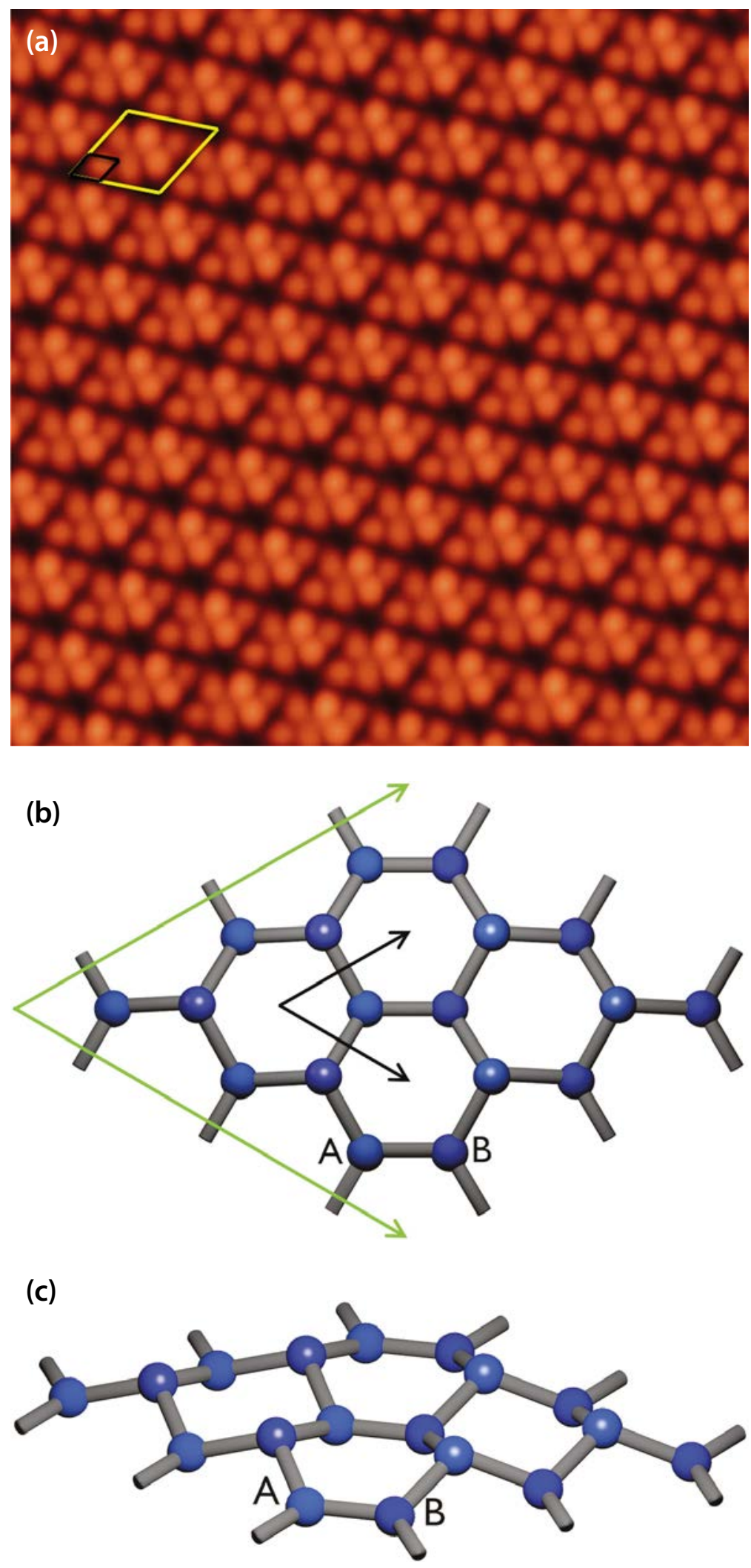

$\triangle$ FIG. 2: Top panel (a): Scanning tunneling microscopy image $(10 \mathrm{~nm} \times 10 \mathrm{~nm}$, tunnel current $0.55 \mathrm{nA}$, sample bias $-520 \mathrm{mV}=$ filled states) showing the observed "flower pattern" resulting from the protruding Si atoms within the $3 \times 3$ reconstructed silicene layer; the $3 \times 3$ supercell is indicated in yellow, while the primitive $1 \times 1$ cell is shown in black. Middle and bottom panels (b) and (c): Top and Perspective views of the geometrical model of the $3 \times 3$ reconstructed silicene layer, as extracted from the $\mathrm{Ag}(111)$ surface where it lies in a $4 \times 4$ coincidence supercell. In the middle panel the $3 \times 3$ supercell vectors are indicated in green, while the basis vectors of the primitive unit cell are indicated in black. 


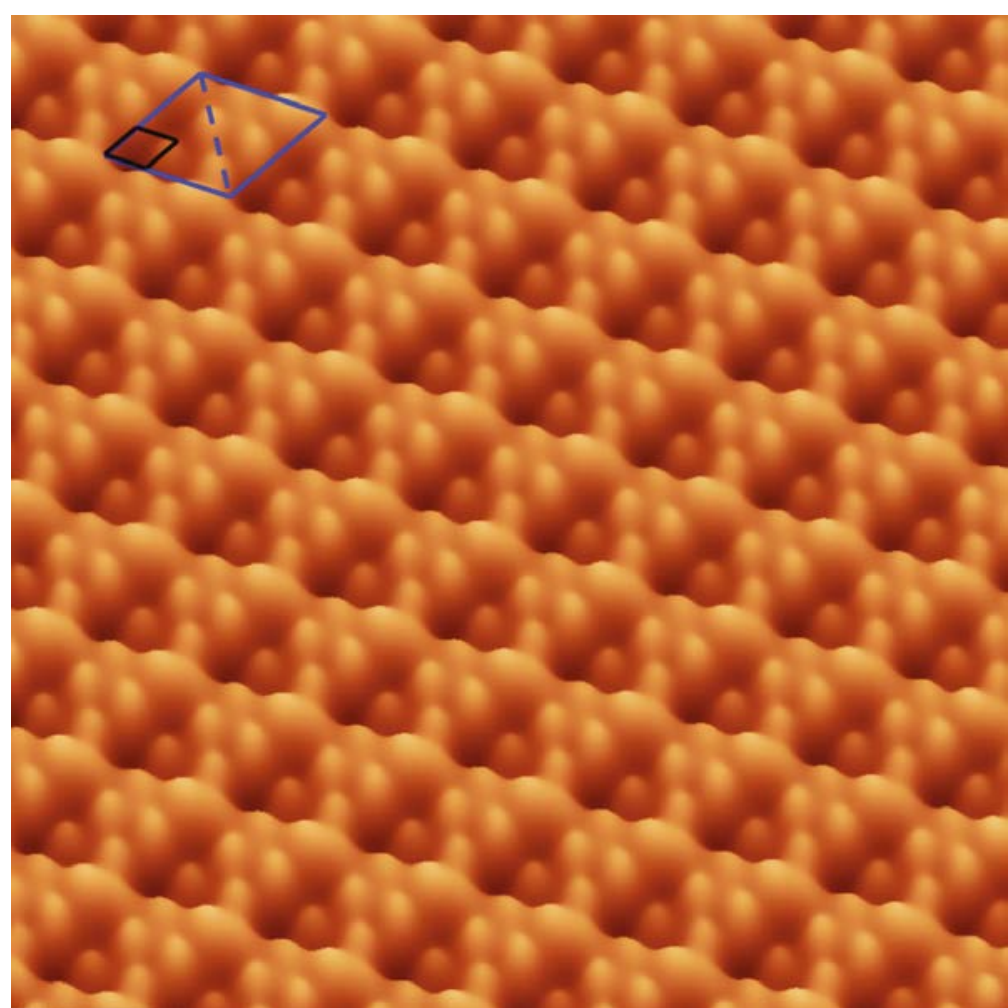

$\Delta$ FIG. 3: STM image $(9 \mathrm{~nm} \times 9 \mathrm{~nm}, 0.33 \mathrm{nA},-200 \mathrm{mV}=$ filled states, 3D rendering $)$ of an ordered, hydrogenated, single layer silicene on $\mathrm{Ag}(111)$. The observed protrusions correspond to adsorbed $\mathrm{H}$ atoms. Although a $3 \times 3$ supercell is preserved (shown in blue, while the primitive $1 \times 1$ is in black), the $\mathrm{H}$ atoms saturate the Si dangling bonds in a manner that favors one of the sublattices ( $6 \mathrm{H}$ atoms on one sublattice the right half of the supercell) over the other (a single $\mathrm{H}$ atom on the other sublattice on the left half of the supercell).

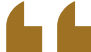

\section{A number of fascinating applications can be envisaged for this robust Dirac 2D material 7}

VFIG. 4: Left: $9 \mathrm{~nm} \times 9 \mathrm{~nm}$ STM image $(0.16 \mathrm{nA},-560 \mathrm{mV}=$ filled states $)$ of the $\sqrt{3} \times \sqrt{3}$ reconstructed surface of multilayer silicene (the $\sqrt{3} \times \sqrt{3}$ supercell is indicated in blue).

Right: Evidence of Dirac fermions in multilayer silicene. A Dirac cone with $\pi$ and $\pi^{*}$ states, below and above the Dirac point, respectively, is recorded in SR-ARPES at the center of the Brillouin zone due to the band folding associated with the reconstruction. The Dirac point is situated at $\sim 0.3 \mathrm{eV}$ below the Fermi level, $\left(\mathrm{E}_{\mathrm{F}}=0.0 \mathrm{eV}\right)$.
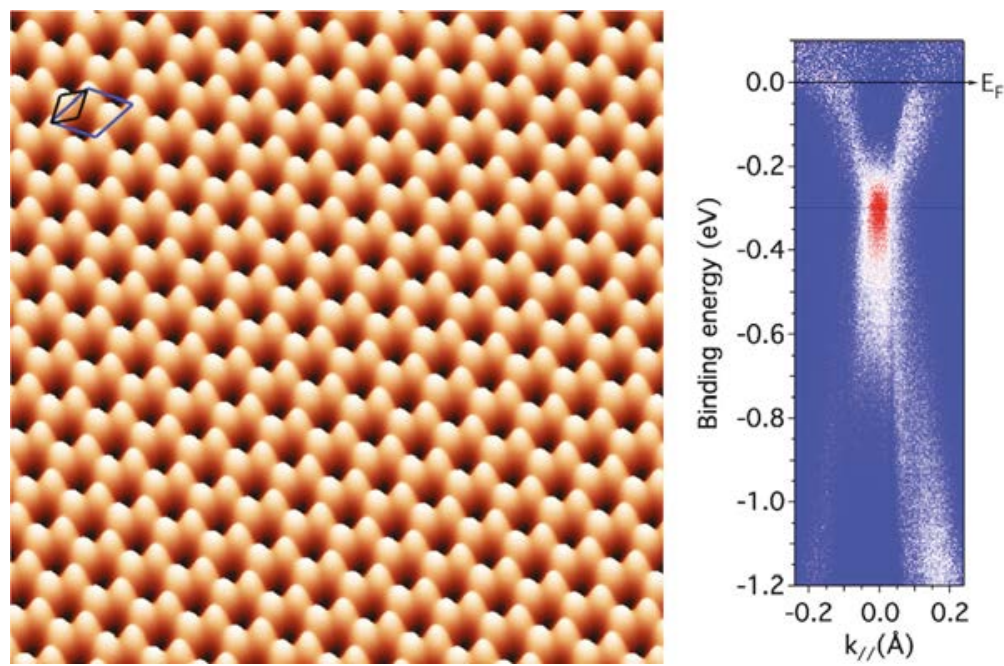

This points to practical applications in electronics, which, as a matter of fact, were already demonstrated with the fabrication of the first silicene FETs operating at room temperature by the groups of Deji Akiwande in Austin (Texas) and Alessandro Molle in Milan (Italy) [13]. This fabrication was made possible by a very smart fabrication process. The tuning of a sizeable gap is, indeed, on the wish list to push Moore's law beyond the $5 \mathrm{~nm}$ node where 2D material channels will become indispensable [14].

\section{Prospects}

The strong spin-orbital coupling in silicene, germanene and stanene gives these novel allotropes a $2 \mathrm{D}$ topological insulator nature that could sustain a robust quantum spin Hall effect at accessible temperatures (in contrast to graphene) [15]. This would hold particularly exciting promises for spintronics and quantum computing.

Beyond the first layer in direct contact with the substrate, we can grow in a successive terrace fashion silicene multilayers, which all possess the same and unique reconstruction $\left(\mathrm{a} 30^{\circ}\right.$ rotated $\sqrt{3} \times \sqrt{3}$ superstructure with respect to the primitive silicene cell) where protruding $\mathrm{Si}$ atoms form the nice honeycomb arrangement seen in Fig. 4 (left). Multilayer silicene hosts Dirac fermions, as evidenced by the measured ARPES cone shown in Fig. 4 (right); here the Dirac point is located at $\sim 0.3 \mathrm{eV}$ below the Fermi level because of charge transfer from the metallic silver substrate yielding partial filling of the $\pi^{*}$ upper cone [16].

While single layer silicene cannot stand air exposure for more than a few minutes (still long enough to measure the transistor characteristics, as indicated above), multilayer silicene is resistant for at least 24 hours: covered by an ultra-thin native oxidized skin, the rest of the body underneath is fully protected [17]. Clearly, a number of fascinating applications can be envisaged for this robust Dirac 2D material.

\section{Conclusion}

We have surveyed only few fascinating properties of single and multilayer silicene, created in 2012, just four years ago, during an 'adventure through the looking glass'. Two years later, single-layer germanene was synthesized on a gold plate [18], while multilayer germanene, which also hosts Dirac fermions, is just born [19]. Indeed, as both silicene and germanene are expected to be easily incorporated into the existing silicon-based industry, their recent synthesis excites a rapidly expanding community.

Furthermore, stanene has just made its debut [20].All these emerging artificial elemental 2D materials, whose properties and potential applications are barely explored, are promised a bright future as they will offer exciting perspectives for future nanoelectronics, spintronics and for quantum computing. Will one of them be the next silicon? At this stage, we think that silicene, i.e., the novel $2 \mathrm{D}$ silicon allotrope, can most probably win the race. 


\section{About the authors}
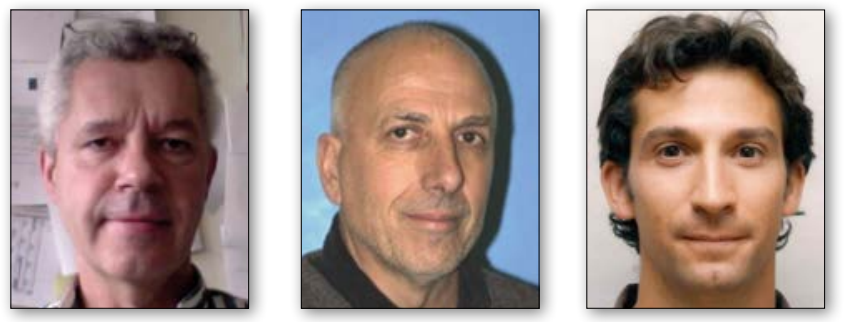

Thierry Angot (left), Guy Le Lay (middle) and Eric Salomon (right) are professors in physics at Aix-Marseille University, in Marseille, France. They are surface/nano scientists, whose interests are in plasma surface interactions (in liaison with the ITER project in Cadarache), semiconductors, organics, and, especially 2D materials. They study both atomic and electronic structures of these novel materials, created in situ, with scanning probe microscopy/spectroscopy and synchrotron radiation based diffraction and spectroscopy advanced methods.

\section{References}

[1] N.M.R. Peres, Europhysics News 40-3, 17 (2009).

[2] B. Radisavljevic, A. Radenovic, J. Brivio, V. Giacometti, and A. Kis, Nature Nanotechnology 6, 147 (2011).

[3] K. Takeda and K. Shiraishi, Phys. Rev. B 50, 14916 (1994).

[4] K.-H. Wu, Chin. Phys. B 24, 086802 (2015)

[5] P. Vogt, P. De Padova, C. Quaresima, J. Avila, E. Frantzeskakis, M. C. Asensio, A. Resta, B. Ealet, and G. Le Lay, Phys. Rev. Lett. 108, 155501 (2012).

[6] A. C. Ferrari et al., Nanoscale 7, 4598 (2015); Unfortunately, silicene on $\mathrm{Ag}(111)$ is naively represented in Fig. 53 by a primitive, nearly flat, honeycomb arrangement, an error to be corrected in the next editions.

[7] R. Hoffmann, Angew. Chem. Int. Ed. 52, 93 (2013).

[8] G. Le Lay, Surface Sci. 132, 169 (1983).

[9] S. Cahangirov, M. Topsakal, E. Aktürk, H. Sahin, and S. Ciraci, Phys. Rev. Lett. 102, 236804 (2009).

[10] Y. Fukaya, I. Mochizuki, M. Maekawa, K. Wada, T. Hyodo, I. Matsuda, and A. Kawasuso, Phys. Rev. B 88, 295413 (2013).

[11] A. Fleurence, R. Friedlein, T. Ozaki, H. Kawai, Y. Wang, and Y. Yamada-Takamura, Phys. Rev. Lett. 108, 245501 (2012).

[12] J. Qiu, H. Fu, Y. Xu, A. I. Oreshkin, T. Shao, H. Li, S. Meng, L. Chen, and K. Wu, Phys. Rev. Lett. 114, 126101 (2015).

[13] L. Tao, E. Cinquanta, D. Chiappe, C. Grazianetti, M. Fanciulli, M. Dubey, A. Molle and D. Akinwande, Nature Nanotechnology 10, 227 (2015).

[14] F. Schwierz, J. Pezoldt and R. Granzer, Nanoscale 7, 8261 (2015).

[15] M. Ezawa, Phys. Rev. B 87, 155415 (2013).

[16] P. De Padova, J. Avila, A. Resta, I. Razado-Colambo, C. Quaresima, C. Ottaviani, B. Olivieri, T. Bruhn, P. Vogt, M. C. Asensio and G. Le Lay, J. Phys.: Condens. Matter 25, 382202 (2013).

[17] P. De Padova, C. Ottaviani, C. Quaresima, B. Olivieri, P. Imperatori, E. Salomon, T. Angot, L. Quagliano, C. Romano, A. Vona, M. Muniz-Miranda, A. Generosi, B. Paci and G. Le Lay, 2D Materials 1, 021003 (2014)

[18] M. E. Dávila, L. Xian, S. Cahangirov, A. Rubio and G. Le Lay, New Journal of Physics 16, 095002 (2014).

[19] M. E. Dávila and G. Le Lay, Sci. Rep. in press.

[20] F.-F. Zhu, W.-J. Chen, Y. Xu, C.-I. Gao, D.-D. Guan, C.-H. Liu, D. Qian, S.-C. Zhang and J.-F. Jia, Nature Materials 14, 1020 (2015). 